\title{
Simvastatin is effective in killing the radioresistant breast carcinoma cells
}

\author{
Bertram Aschenbrenner ${ }^{1,2,3}$, Giulia Negro ${ }^{1,2,3}$, Dragana Savic ${ }^{1,2,3}$, Maxim Sorokin ${ }^{3,4,5,6}$, \\ Anton Buzdin ${ }^{3,6,7,8}$, Ute Ganswindt ${ }^{1}$, Maja Cemazar ${ }^{3,9}$, Gregor Sersa ${ }^{9}$, Sergej Skvortsov ${ }^{1,2}$, \\ Ira Skvortsova ${ }^{1,2,3}$
}

\author{
${ }^{1}$ Medical University of Innsbruck, Therapeutic Radiology and Oncology, Innsbruck, Austria \\ ${ }^{2}$ Tyrolean Cancer Research Institute, Innsbruck, Austria \\ ${ }^{3}$ EORTC PathoBiology Group \\ ${ }^{4}$ Institute of Personalized Medicine, Sechenov First Moscow State Medical University, Moscow, Russia \\ ${ }^{5}$ Omicsway Corp., Walnut, USA \\ ${ }^{6}$ Shemyakin-Ovchinnikov Institute of Bioorganic Chemistry, Moscow, Russia \\ ${ }^{7}$ Oncobox ltd., Moscow, Russia \\ ${ }^{8}$ World-Class Research Center "Digital Biodesign and Personalized Healthcare", Sechenov First Moscow State Medical \\ University, Moscow, Russia \\ 9 Institute of Oncology Ljubljana, Department of Experimental Oncology, Ljubljana, Slovenia
}

Radiol Oncol 2021; 55(3): 305-316.

Correspondence to: Prof. Ira Skvortsova, Laboratory for Experimental and Translational Research on Radiation Oncology (EXTRO-Lab), Department of Therapeutic Radiology and Oncology, Medical University of Innsbruck, Anichstr. 35, A-6020 Innsbruck, Austria.

E-mail: Ira.Skvortsova@i-med.ac.at

Disclosure: No potential conflicts of interest were disclosed.

This is an open access article under the CC BY-NC-ND license (http://creativecommons.org/licenses/by-nc-nd/4.0/).

Background. Statins, small molecular 3-hydroxy-3-methylglutaryl-coenzyme A reductase inhibitors, are widely used to lower cholesterol levels in lipid-metabolism disorders. Recent preclinical and clinical studies have shown that statins exert beneficial effects in the management of breast cancer by increasing recurrence free survival. Unfortunately, the underlying mechanisms remain elusive.

Materials and methods. Simvastatin, one of the most widely prescribed lipophilic statins was utilized to investigate potential radiosensitizing effects and an impact on cell survival and migration in radioresistant breast cancer cell lines. Results. Compared to parental cell counterparts, radioresistant MDA-MB-231-RR, T47D-RR andAu565-RR cells were characterized by upregulation of 3-hydroxy-3-methylglutharyl-coenzyme A reductase (HMGCR) expression accompanied by epithelial-to-mesenchymal transition (EMT) activation. Radioresistant breast cancer cells can be killed by simvastatin via mobilizing of a variety of pathways involved in apoptosis and autophagy. In the presence of simvastatin migratory abilities and vimentin expression is diminished while E-cadherin expression is increased.

Conclusions. The present study suggests that simvastatin may effectively eradicate radioresistant breast carcinoma cells and diminish their mesenchymal phenotypes.

Key words: breast cancer cells; cancer stem cells; radiotherapy; migration; simvastatin

\section{Introduction}

Breast cancer is the most diagnosed malignant tumor in women. It is estimated over 355,000 cases in the European Union will be registered in 2020 that corresponds to $13.3 \%$ of all diagnosed malignan- cies. ${ }^{1}$ Therapeutic management of breast cancer is markedly changed during last 20 years. Thus, after breast-conserving surgery, all patients receive either partial or whole-breast radiation therapy. ${ }^{2}$ This strategy allows to significantly reduce the risk of local breast cancer recurrences and breast can- 
cer-related mortality. ${ }^{3,4}$ However, ipsilateral local recurrences still occur in locally and systemically treated breast cancer patients. Current clinical classification requires to determine whether an ipsilateral relapsed tumor is a true recurrence or a second primary tumor. 5,6 The relapsed tumors and second primary tumors are distinct in their localization and molecular features. It was also found that recurrence-free and overall survival rates for true recurrences are significantly shorter than for the second primary tumors. ${ }^{7}$ We, therefore, assumed that true recurrences usually occur from surviving breast carcinoma cells after primary treatment using radiotherapy with or without chemotherapeutics, antihormonal or targeted agents. The cells recovered after their exposure to anti-cancer therapeutic approaches may possess a treatment resistant phenotype characterized by enhancement of pro-survival mechanisms protecting them from cytotoxic or cytostatic agents. It is logical to suggest that carcinoma cells comprising the tumor recurrences can demonstrate radiation resistance due to their abilities to survive and further proliferate within the irradiated field. To have a therapeutic benefit from re-irradiation, the radiation dose should be markedly increased to effectively kill radioresistant breast carcinoma cells. Unfortunately, due to the problem of tissue tolerance, the required dose can usually not be achieved $^{3}$, and a lower dose of re-irradiation is not able to successfully eradicate radioresistant carcinoma cells and can even increase the molecular characteristics underlying radiation resistance.

Since therapy resistant carcinoma cells can also possess an augmentation of their metastatic capacities, true local breast cancer recurrences are often accompanied by distant metastasis. ${ }^{8,9}$ These metastatic lesions can also be treated by radiotherapy. However, an efficacy of this therapeutic approach might be diminished due to radiation resistance of breast carcinoma cells spreading to distant organs and tissues. Therefore, it is logical to suggest the use of systemic treatment to improve radiation response and combat metastatic spread of radioresistant breast carcinoma cells.

Systemic treatment of cancer can include a variety of different agents. Our research group has focused on the lipid-lowering drug simvastatin, which is often used by breast cancer patients independently from their cancer diagnose. Simvastatin as other statins is a competitive inhibitor of 3-hydroxy-3-methylglutharyl-coenzyme A reductase (HMGCR). Although anti-cancer activity of statins is described in literature ${ }^{10}$, there is still no agree- ment whether statins generally and simvastatin particularly can be used to improve therapy response of radioresistant breast carcinoma cells, and which molecular properties of carcinoma cells make them susceptible to simvastatin treatment.

Therefore, the main aim of this study was to determine the sensitivities of radioresistant breast carcinoma cells to simvastatin and mechanisms underlying the cellular responses to the drug alone or in combination with ionizing radiation.

\section{Materials and methods}

\section{Cell culture and treatment with ionizing radiation}

MDA-MB-231 (triple-negative type: estrogen, progesterone and HER2 receptor negative (ER-, PR-, HER2-), T47D (luminal A type: ER+, PR+, HER2) and Au565 (Her2-positive type: ER-, PR-, HER2/ neu+) cells were purchased from the American Type Culture Collection. All cell lines were grown in RPMI1640 medium supplemented with $2 \mathrm{mM}$ L-glutamine, $50 \mathrm{U} / \mathrm{mL}$ penicillin, $50 \mu \mathrm{g} / \mathrm{mL}$ streptomycin (Thermo Fisher Scientific, Vienna, Austria), and $10 \%$ fetal bovine serum (FBS) $\left(\right.$ HyClone $^{\mathrm{Tm}}$ ) (Thermo Fisher Scientific, Vienna, Austria). T47D cells were maintained in medium containing $10 \mu \mathrm{g} /$ $\mathrm{ml}$ bovine insulin (Sigma Aldrich, Millipore Merck, Vienna, Austria). Cell cultures were incubated in a $5 \% \mathrm{CO}_{2}$ humidified atmosphere at $37^{\circ} \mathrm{C}$.

Radiation-resistant cells, MDA-MB-231-RR, T47D-RR and Au565-RR (RR cells), were obtained from parental breast cancer cells after repetitive exposure to ionizing radiation (10 Gy) (16 MV x-rays) using an Elekta Precise Linear Accelerator (Elekta Oncology Systems, UK) at a dose rate of approximately $1.8 \mathrm{~Gy} / \mathrm{min}$. The cells were irradiated every 2 weeks when breast cancer cells recovered from their exposure to ionizing radiation. Cells which survived after irradiation (total dose of $100 \mathrm{~Gy}$ ) were collected for further experiments. The newly received cell lines maintained resistance to ionizing radiation independently from a number of passages.

Simvastatin was purchased from Calbiochem (Merck Millipore, Vienna, Austria). Simvastatin was dissolved in dimethyl sulfoxide (DMSO, Sigma Aldrich, Merck Millipore, Vienna, Austria) and used at a final clinically relevant concentration of $8 \mu \mathrm{M}$.

Cells were irradiated at single doses of 2, 4, 6, and $8 \mathrm{~Gy}$ to determine radiation response of parental and RR breast carcinoma cells, and at a single 
clinically relevant dose of 2 Gy for all other experiments using an Elekta Precise Linear Accelerator (Elekta Oncology Systems, UK).

\section{D tomographic microscopy}

Parental and radioresistant MDA-MB-231, T47D, and Au565 cells were seeded into the glassbottom dishes with a diameter of $35 \mathrm{~mm}$ (Ibidi, Switzerland), and cells were incubated for 24 hours at $37^{\circ} \mathrm{C}$ and $5 \% \mathrm{CO}_{2}$ humidified atmosphere. Next, cells were analysed for their morphology using 3D tomographic microscope with a $60 x$ objective (3D Cell Exlplorer-FLUO, Nanolive SA, Switzerland), and 3D tomographic images (z-stacks) were collected.

\section{Apoptosis assay}

Investigated breast carcinoma cells (parental and radioresistant MDA-MB-231, T47D and Au565) were seeded and cultured overnight in 6-well plates at $1,0 \times 10^{\wedge} 5$ cells/well. Cells were exposed to ionizing radiation at single doses of $0,2,4,6$, and 8 Gy. After being cultured for 72 hours, irradiated cells were collected for apoptosis assay. Briefly, the cells were trypsinized and then pelleted by centrifugation at $300 \mathrm{~g}$ for 10 minutes at $4^{\circ} \mathrm{C}$. The supernatant was discarded, and the pellet was washed once with cold PBS and further resuspended in Annexin- $\mathrm{V}$ binding buffer containing Annexin- $\mathrm{V}$ APC and propidium iodide (PI) (AnxA100PI Kit, MabTag, Friesoythe, Germany). Cells were stained in darkness for 15 minutes, and the same volume $(100 \mu \mathrm{L})$ of Annexin-V binding buffer was added to each sample and the prepared samples were analyzed by flow cytometry (BD FACSCantoTM II). The percentage of Annexin- $\mathrm{V}$ and PI positive cells was evaluated using the FlowJo_V10.6.2 software. Three independent experiments in duplicates were performed.

\section{Cell death development and Sub-G1 evaluation}

Breast carcinoma cells were seeded in 6-well plates and treated either with DMSO alone as a vehicle control, simvastatin $(8 \mu \mathrm{M})$ alone, irradiation alone (2 Gy) or combination treatment using cell pretreatment with simvastatin $(8 \mu \mathrm{M})$ for 24 hours followed by irradiation (2 Gy). Cell death development was studied during 72 hours after simvaststin treatment and different time points of 24 hours, 48 hours and 72 hours were selected for analysis.
To evaluate the induction of cell death, samples were analyzed as previously described. ${ }^{11}$ Briefly, all cells were harvested at the indicated time points followed by centrifugation for 10 minutes $(300 \mathrm{~g})$ at $4^{\circ} \mathrm{C}$. The pellets were washed with cold PBS and resuspended in hypotonicfluorochrome solution $(50 \mu \mathrm{g} / \mathrm{mL}$ propidium iodide (PI), $0.1 \%$ sodium citrate, $0.1 \%$ Triton $\mathrm{X}-100)$. The samples were stained in darkness for 30 minutes at $4^{\circ} \mathrm{C}$ followed by flow cytometry (BD FACSCantoTM II) analysis. To determine DNA fragmentation, PI fluorescence of individual nuclei was evaluated with an excitation wavelength of $488 \mathrm{~nm}$ and an emission wavelength of $670 \mathrm{~nm}$. Gating was done on single nuclei to exclude doublets and debris from the analysis. Cell cycle analysis was performed using FlowJo_V10.6.2 software, and the Sub-G1 fraction was determined.

\section{Western blot analysis}

Western blot was performed as published previously $^{12,13}$ using E-cadherin, caspase-3, caspase-7, caspase-8, caspase-9, PARP-1, cytochrome C, XIAP, AIF, beclin-1, LC3 A/B rabbit monoclonal antibody (Cell Signaling Technology, Inc., Beverly, MA, USA), HMGCR rabbit monoclonal antibody (Abcam, UK), Vimentin, Smac/DIABLO mouse (Cell Signaling Technology, Inc., Beverly, MA, USA). Loading control was evaluated using $\alpha$-tubulin rabbit monoclonal antibody (Cell Signaling Technology, Inc., Beverly, MA, USA). For evaluation of protein expression, X-ray films (GE Healthcare, Chicago, IL, USA) were scanned and analyzed by the Image StudioTM Lite 5.0 (LI-COR Biotechnology, Lincoln, NB, USA). The Integrated Density Value (IDV) was obtained as a ratio of normalized protein band densities in parental and radioresistant RR cells after background correction.

\section{Migration assay}

Scratch assay or wound healing assay was performed to evaluate two-dimensional cancer cell migration. Parental and radioresistant breast carcinoma cells were grown to confluence in 6-well plates. A scratch was made on the monolayer using a sterile $200 \mu \mathrm{L}$-pipette tip. The monolayer was rinsed three times with PBS and placed in the appropriate complete medium with either simvastatin dissolved in DMSO $(8 \mu \mathrm{M})$ or DMSO as a vehicle control. Phase contrast images were made during 20 hours at a magnification of $4 x$ (Lionheart Live Cell Microscope, BioTek, Bad Friedrichshall, 

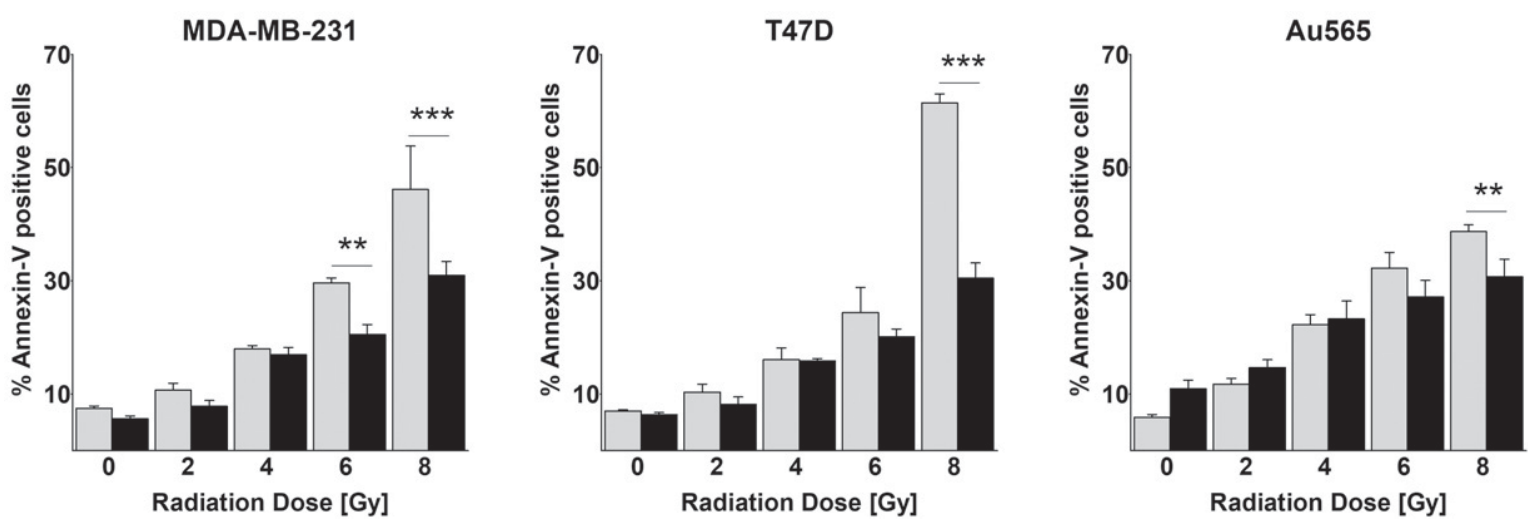

FIGURE 1. Radiation-induced apoptosis in breast carcinoma cells. Radiation sensitivity of the investigated parental and radioresistant breast carcinoma cells was determined using apoptosis assay as described in the section Materials and methods. Grey bars represent parental cells and black bars the radioresistant cells. All experiments were performed at least three times in duplicates; $*=p<0.05 ; * *=p<0.01 ; * * *=p<0.001$.

Germany), and the gap width was measured using the Gen5 (V. 3.08) software. The percentage of the gap width which remained after 20 hours of cell incubation was plotted.

\section{Statistical analysis}

GraphPad Prism software was used to plot the graphs and for statistical evaluation. All the values are represented as the means \pm standard error of the mean. Statistical comparisons were performed by one-way analysis of variance (ANOVA) followed by Bonferroni post-hoc comparisons to analyze the differences between each group. Statistical significance was defined as: ${ }^{*}=\mathrm{P} \leq 0,05,{ }^{*}=\mathrm{P} \leq$ $0,01,{ }^{* * *}=\mathrm{P} \leq 0.001$ and $^{* * * *}=\mathrm{P} \leq 0.0001$.

\section{Results}

\section{Radiation resistance of breast carcinoma cells}

To confirm that newly received breast carcinoma cells (RR cells) possess radiation resistance, parental and RR cells were exposed to different doses of ionizing radiation and their susceptibility to apoptosis was determined. As it is seen in Figure 1, all three MDA-MB-231-RR, T47D-RR, and Au565-RR cells were less sensitive to irradiation than their parental counterparts. Although there are no significant differences in apoptosis development in parental and RR cells irradiated at lower single doses (2 and $4 \mathrm{~Gy}$ ), cell exposure to ionizing radiation at higher doses of 6 and 8 Gy was accompanied by more pronounced cell death in parental cells. Thus, irradiation at a dose of $8 \mathrm{~Gy}$ induced $30.93 \pm 2.47 \%$ AnnexinV-PI-positive cells in MDA-MB-231-RR, $30.46 \pm 2.71 \%$ in T47D-RR, and $30.75 \pm 3.08 \%$ in Au565-RR cells versus $46.15 \pm 7.67 \%, 61.40 \pm 1.60 \%$, and $38.69 \pm 1.22 \%$ in parental MDA-MB-231, T47D, and Au565 breast carcinoma cells, respectively.

\section{HMGCR expression in breast carcinoma cells}

Since HMGCR is a target for simvastatin, its expression was evaluated in parental and RR breast carcinoma cells (Figure 2A). It was found that triple-negative MDA-MB-231-RR and hormone receptor positive T47D-RR cells were characterized by up-regulation of HMGCR in comparison with their parental counterparts. It is necessary to note that parental T47D breast carcinoma cells did not express HMGCR whereas T47D-RR cells showed HMGCR overexpression. Surprisingly, Her2positive Au565-RR demonstrated slight downregulation of HMGCR compared to the parental Au565 cells.

Administration of simvastatin alone at a clinically relevant doses of $8 \mu \mathrm{M}$ resulted in significant downregulation of HMGCR in all investigated breast carcinoma cells (Figure 2B). Cell exposure to the clinically relevant single dose of irradiation of 2 Gy led to the substantial increase of HMGCR expression especially at 24 hours after the treatment. Simvastatin-pretreated parental and RR breast carcinoma cells were protected from radiation-induced HMGCR upregulation, which did not significantly differ from those in cells treated with simvastatin alone. 
(A)

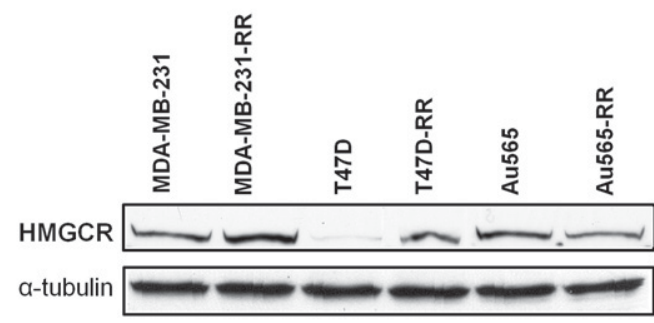

HMGCR expression

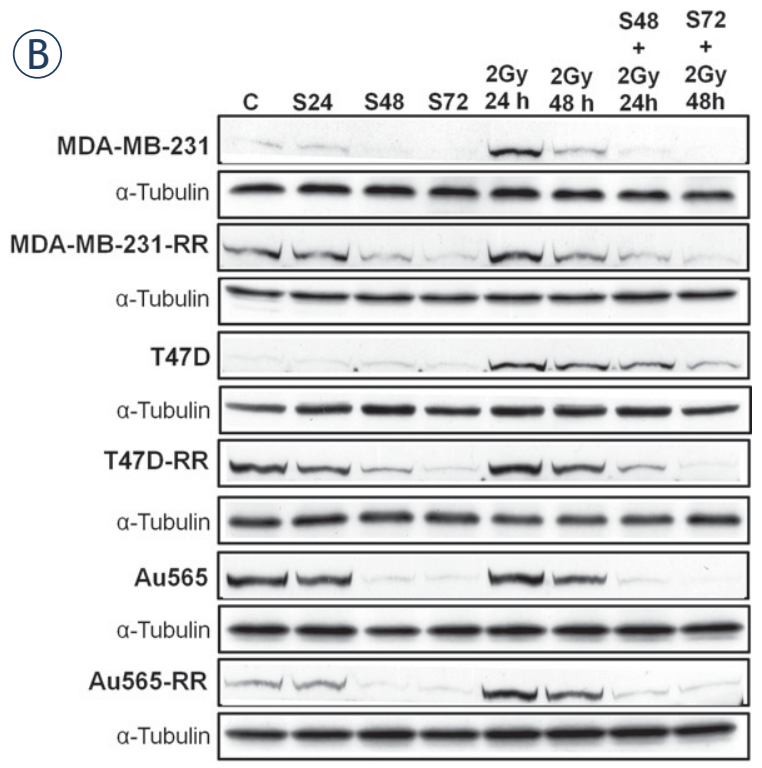

FIGURE 2. HMGCR expression in breast carcinoma cells. (A) Constitutive 3-hydroxy-3-methylglutharyl-coenzyme A reductase (HMGCR) expressions in parental and radioresistant breast carcinoma cells. Protein extracts from total cell lysates were subjected to Western blot analysis, and constitutive levels of HMGCR were determined in all investigated breast carcinoma cells; (B) Simvastatin-caused modulation of HMGCR expression in parental and radioresistant breast carcinoma cells was confirmed using Western blot analysis.

\section{Simvastatin-caused modulation of migratory abilities of breast carcinoma cells}

Next, we have investigated whether RR breast carcinoma cells are altered in their migratory capacities (Figure 3A, 3B). Triple-negative MDA-MB231-RR and hormone receptor-positive T47D-RR breast carcinoma cells showed increased migratory properties compared to their parental counterparts. Using scratch assay, it was observed that MDA-MB-231-RR cells were able to close the gap within 20 hours, whereas parental MDA-MB-231 cells demonstrated $51.62 \pm 2.55 \%$ of the original gap width left at this time point. Very similar data
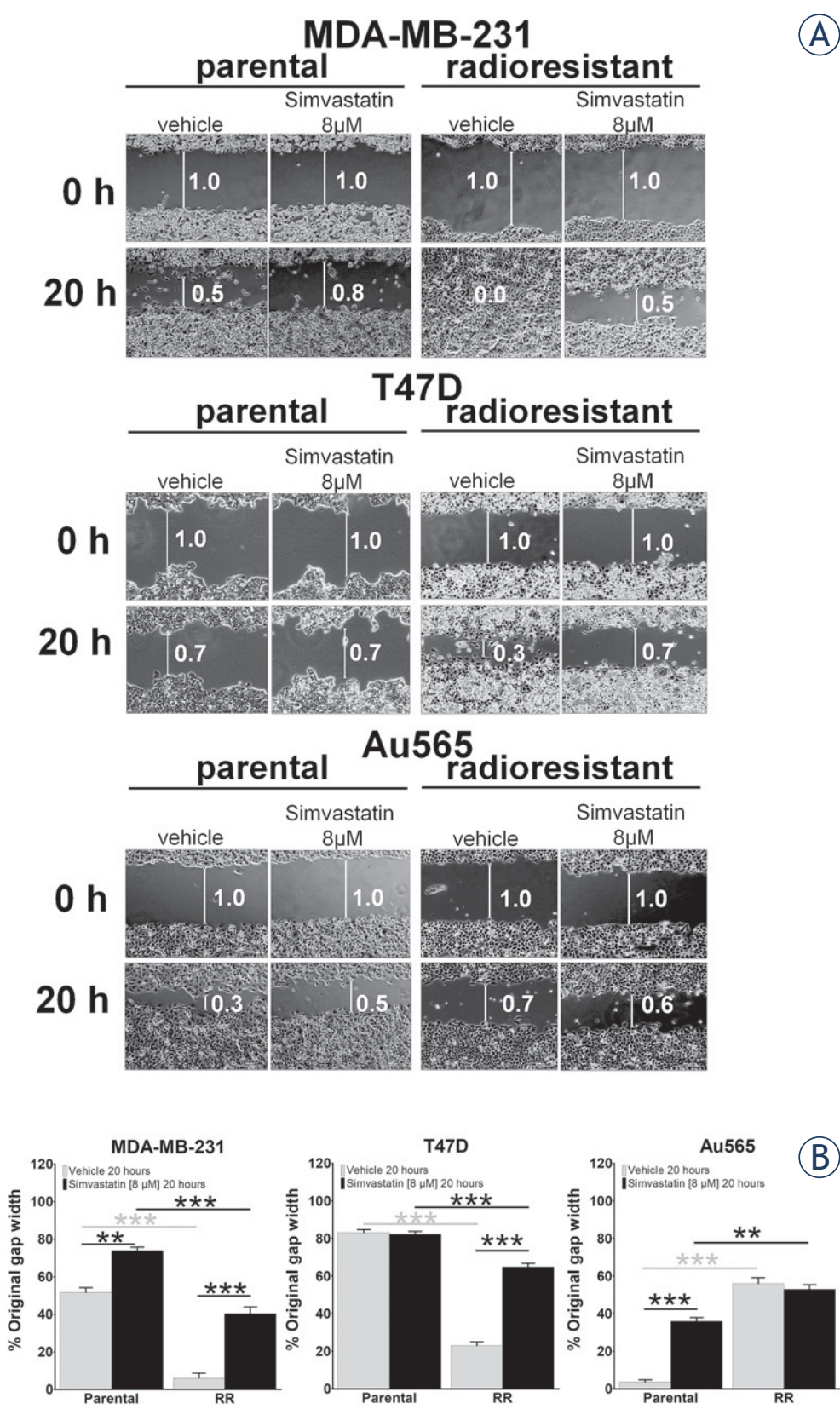

FIGURE 3. Simvastatin-regulated breast carcinoma cell migration. (A) Wound healing assay was used to determine how simvastatin affected breast carcinoma cell migration. Cell migration was assayed at a magnification of $4 x$ (Lionheart Live Cell Microscope, BioTek, Bad Friedrichshall, Germany). The cell migration rates were determined as a ratio between the gap width at indicated time point and initial gap width at 0 hours; (B) Statistical evaluation of the gap width in breast carcinoma cells. Gap width was measured using the Gen5 (V. 3.08) software and the percentage of the gap width remained after 20 hours of cell incubation in presence of DMSO as a vehicle control or simvastatin $(8 \mu \mathrm{M})$ was plotted. All experiments were performed at least three times in duplicates; ${ }^{*}=p<0.05 ;{ }^{* *}=p<0.01 ;{ }^{* *}=p<0.001$.

were received when migratory capacities of T47DRR breast carcinoma cells were compared with those in parental T47D cells. Thus, parental T47D cells had $83.08 \pm 1.71 \%$ of the gap open 20 hours 
(A)

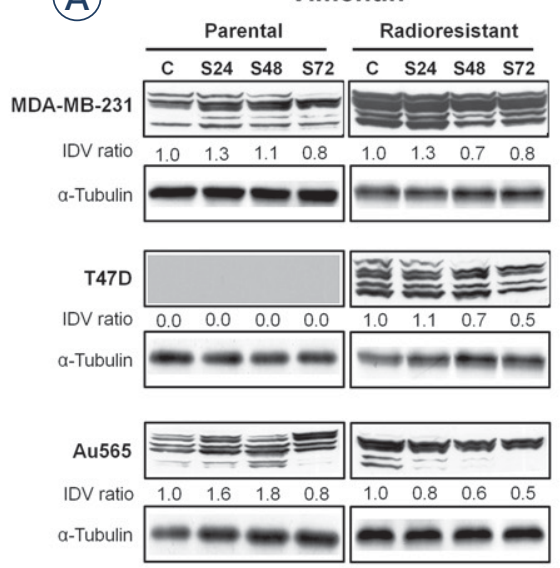

Parental

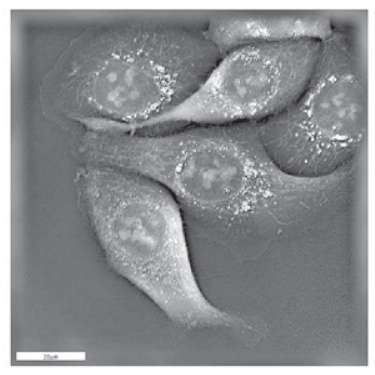

MDA-MB-231

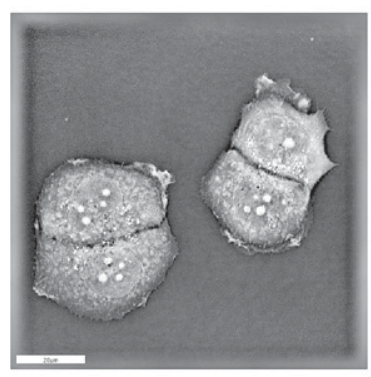

T47D
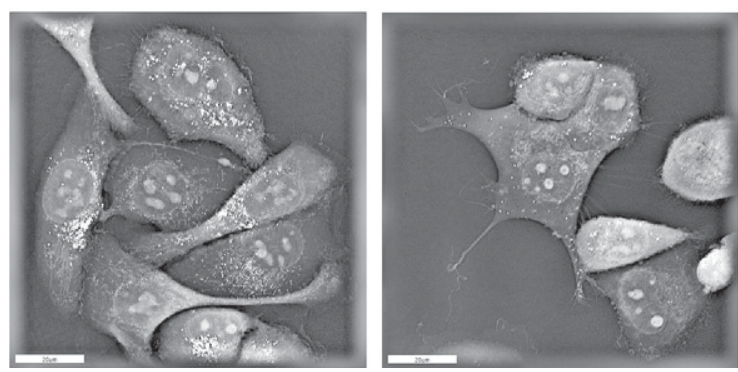

FIGURE 4. Mesenchymal and epithelial markers in breast carcinoma cells treated with simvastatin. (A) Simvastatin-dependent regulation of vimentin and E-cadherin expressions in parental and radioresistant breast carcinoma cells were evaluated using Western blot analysis as described in the section Materials and Methods. IDV was calculated for each protein band and normalized to the a-tubulin band density after background correction. IDV ratio means fold-change of vimentin or E-cadherin band densities in simvastatin-treated compared to the vehicle-treated breast carcinoma cells. (B) 3D holographic breast cancer cell microscopy. Parental and radioresistant MDA-MB-231, T47D, and Au565 cells were analyzed for their morphology using 3D Nanolive Explorer-FLUO as described in the Materials and Methods. after scratching, whereas radioresistant T47D-RR cells demonstrated only $22.97 \pm 2.05 \%$ of the original gap width open. In contrast, parental Her2neu-positive Au565 cells were more migratory than their radioresistant Au565-RR counterparts with the gap closure of more than $\sim 95 \%$ and $~ 60 \%$, respectively.

Simvastatin significantly reduced migratory abilities of parental and radioresistant MDA-MB231-RR, radioresistant T47D-RR, and parental Au565 breast carcinoma cells. The most prominent inhibition of cell migration was observed in radioresistant MDA-MB-231-RR and T47D-RR cells ( 5-fold simvastatin-caused reduction of migration in MDA-MB-231-RR cells versus 1.6-fold in parental MDA-MB-231 cells, and 3-fold simvastatininduced reduction of migration in T47D-RR cells versus no effect observed in simvastatin-treated parental T47D cells). Surprisingly, radioresistant Au565-RR cells were not affected in their migratory capacities by simvastatin, and the gap was equally closed by the untreated and treated Au565-RR cells.

\section{Simvastatin-regulated expression of epithelial and mesenchymal markers in breast carcinoma cells}

Considering that radioresistant breast carcinoma cells were affected in their sensitivity to ionizing radiation and altered in their migratory capacities, we next analyzed the levels of expression of mesenchymal (vimentin) and epithelial (E-cadherin) markers in the investigated carcinoma cells (Figure 4A). It was found that all radioresistant breast carcinoma cells acquired a more mesenchymal phenotype compared to parental cells. Interesting to note that radioresistant MDA-MB231-RR cells lost E-cadherin expression accompanied by up-regulation of vimentin. Parental T47D cells had a pure epithelial phenotype with overexpression of E-cadherin with no sign of vimentin expression, and radioresistant T47D-RR cells showed a switch toward mixed epithelial and mesenchymal phenotype characterized by overexpression of both epithelial and mesenchymal markers. Parental Au565 cells and radioresistant Au565-RR cells were equal in their mesenchymal phenotype with vimentin overexpression and lack of E-cadherin expression. Cell treatment with simvastatin resulted in the time-dependent down-regulation of vimentin and up-regulation of E-cadherin in all investigated breast carcinoma cells. Even the cells lacking E-cadherin revealed a simvastatin-induced up-regulation of the epithelial marker. Epithelial- 
to-mesenchymal transition of radioresistant breast carcinoma cells was accompanied by morphological changes characterized by the membrane ruffling, filopodia and lamellipodia formation resulting in the increased cellular surfaces (Figure 4B).

\section{Cell death development in breast carcinoma cells treated with simvastatin and ionizing radiation}

As previously mentioned, radioresistant breast carcinoma cells are affected in the susceptibility to apoptosis caused by ionizing radiation. Since apoptosis represents only one type of cell death, we decided to be focused on the evaluation of the total treatment-induced cell killing using Nicoletti staining.

Cell death development was determined in all investigated breast cancer cells after their exposure to simvastatin alone, irradiation alone, and their combination. It was observed that among all investigated breast carcinoma cell lines only parental T47D cells were not sensitive to simvastatin (Figure 5). All other parental and radioresistant breast carcinoma cells demonstrated time-dependent cell death development in response to cell exposure to simvastatin at a clinically relevant dose of $8 \mu \mathrm{M}$. Thus, parental MDA-MB-231, radioresistant MDA-MB-231$R R$ and radioresistant T47D-RR cells showed equal cell death development with $46.35 \pm 4.38 \%$, $43.78 \pm$ $3.19 \%$, and $51.35 \pm 2.96 \%$ at 96 hours, respectively. Parental and radioresistant Au565 breast carcinoma cells were less susceptible to simvastatin with cell death of $41.96 \pm 9.05 \%$ for parental Au565 cells and $27.50 \pm 6.03 \%$ for radioresistant Au565-RR cells at 96 hours after simvastatin treatment.

Breast cancer cell exposure to the clinically relevant single dose of irradiation of 2 Gy did not result in the substantial cell death in all investigated breast carcinoma cell lines. Parental and radioresistant Au565 cells were the most radiation sensitive among other cell lines, and radiation-caused cell death was $17.43 \pm 2.58 \%$ in parental Au565 cells and $14.23 \pm 1.38 \%$ in Au565-RR cells. Unfortunately, combination of simvastatin and irradiation did not lead to the enhancement of cell death compared to simvastatin alone in all parental and radioresistant breast carcinoma cells.

\section{Simvastatin activates different types of cell death}

To understand which types of cell death are induced by simvastatin, we have performed Western

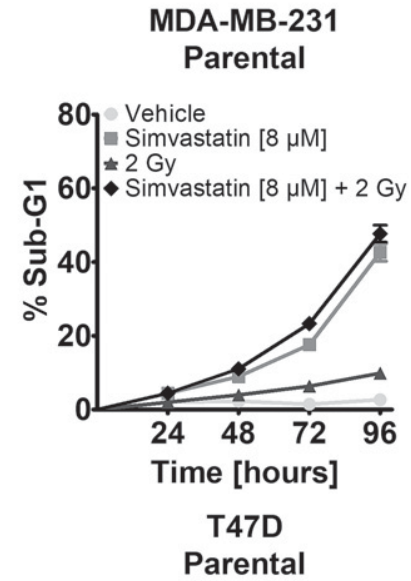

MDA-MB-231

RR

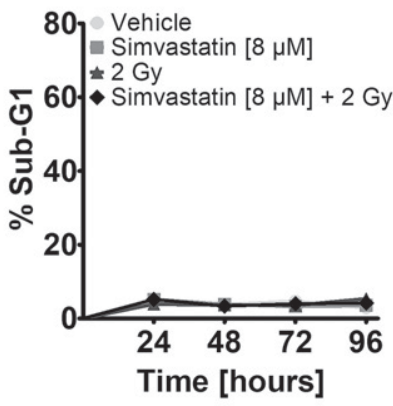

Au565

Parental

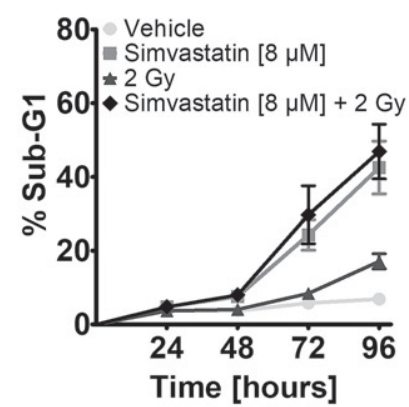

FIGURE 5. Cell death development in breast carcinoma cells treated with simvastatin or irradiation or their combination. At indicated time points, analysis of sub-G1 cell fraction was evaluated in the samples collected after treatment of parental and radioresistant breast carcinoma cells with simvastatin $(8 \mu \mathrm{M})$ alone, irradiation ( $2 \mathrm{~Gy}$ ) alone or combination of simvastatin $(8 \mu \mathrm{M})$ and irradiation (2 Gy). All experiments were performed at least three times in duplicates; ${ }^{*}=p<0.05 ;{ }^{* *}=p<0.01 ;{ }^{* *}=p<0.001$.

blot analysis for the key regulators of caspasedependent, caspase-independent apoptosis and autophagy (Figure 6). Interesting to note that parental and radioresistant triple-negative MDAMB-231, hormone receptor-positive T47D, and Her2neu-positive Au565 breast carcinoma cells demonstrated different mechanisms of cell death development. Both intrinsic and extrinsic apoptosis pathways were implicated in simvastatin-triggered 

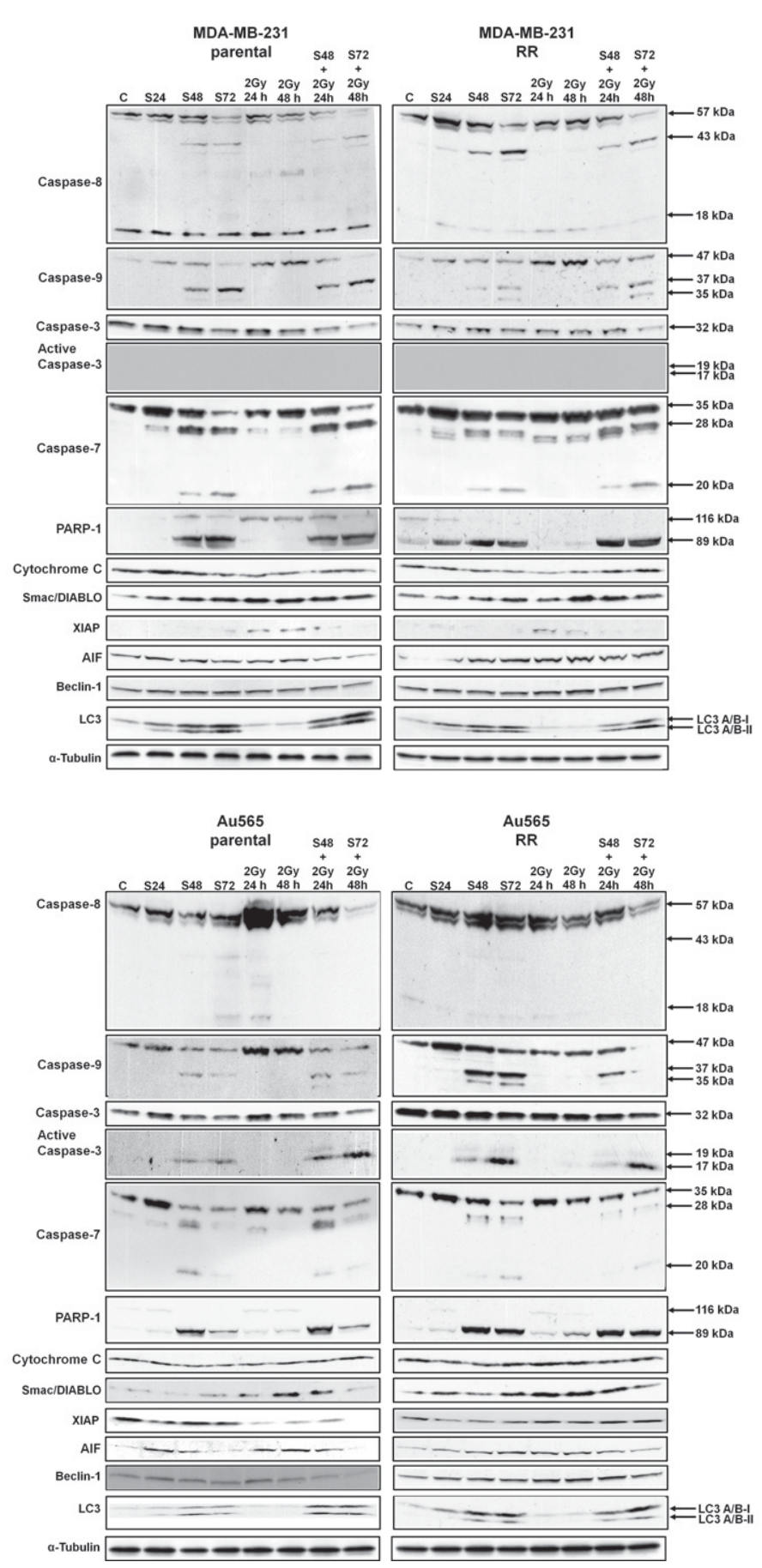

cell death in all investigated breast carcinoma cells. First, it was seen that initiator caspase- 8 belonging to the extrinsic apoptosis pathway was activated in parental and radioresistant MDA-MB-231 cells independently from the kind of cell treatment, and both cleaved forms (43 $\mathrm{kDa}$ and $18 \mathrm{kDa}$ ) were observed. In contrast, all other cell lines were deficient in full caspase- 8 cleavage. Thus, parental

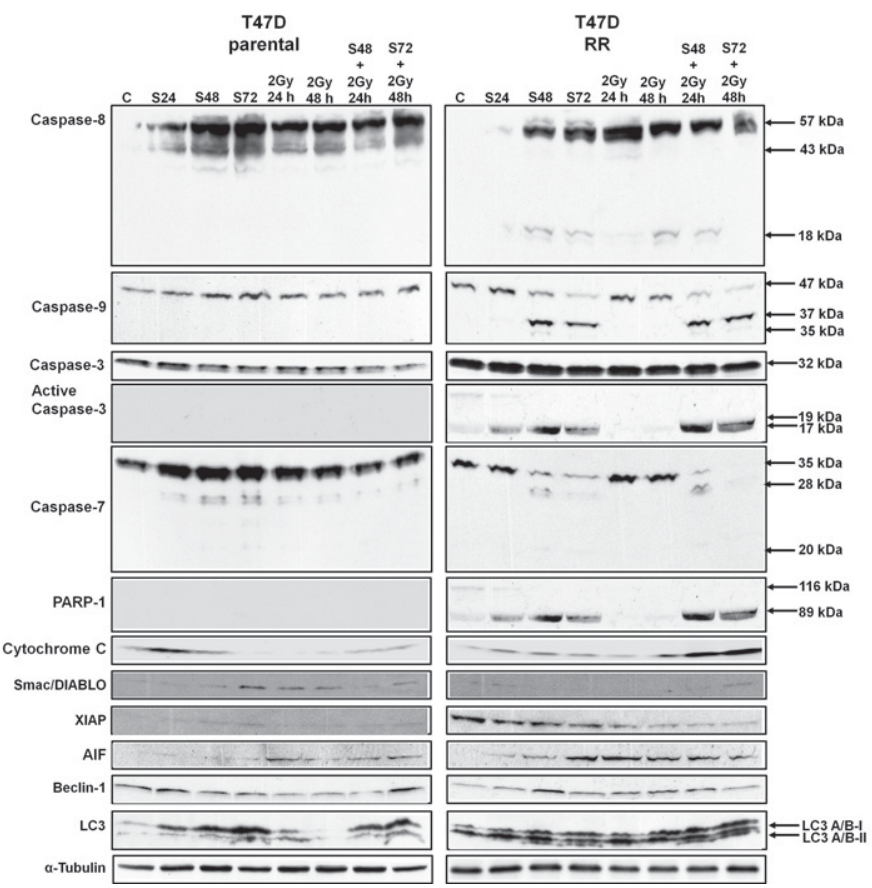

FIGURE 6. Regulation of apoptosis- and autophagy-related proteins in breast carcinoma cells. Treatment-induced modulation of protein expression in parental and radioresistant breast carcinoma cells were investigated using Western blot analysis. Cells were either treated with simvastatin $(8 \mu \mathrm{M})$, or irradiation alone at a dose of $2 \mathrm{~Gy}$, or with combination treatment using simvastatin $(8 \mu \mathrm{M})$ pretreatment (24 hours) followed by irradiation at a single dose of $2 \mathrm{~Gy}$. Protein extractions were performed at the indicated time points, and then samples were analyzed using Western blotting as described in Materials and Methods.

T47D cells demonstrated only one cleaved form (43 $\mathrm{kDa})$ after treatment with simvastatin alone, irradiation alone, or combination treatment, whereas radioresistant T47D-RR cells possessed similar cleavage only after cell treatment with simvastatin alone at 48 and 72 hours, or with the combination treatment (simvastatin 48 hours + irradiation 24 hours). Parental Au565 breast carcinoma cells had full caspase- 8 cleavage after cell exposure to simvastatin alone at 72 hours or irradiation alone at 24 hours. Radioresistant Au565-RR cells did not reveal a pronounced caspase- 8 cleavage after any kind of treatment.

Activation of the intrinsic apoptosis pathway was characterized by caspase- 9 cleavage. In comparison with parental breast carcinoma cells, their radioresistant counterparts revealed a more pronounced caspase-9 cleavage after simvastatinbased treatments. It was manifested either in a more pronounced expression of the cleaved form 

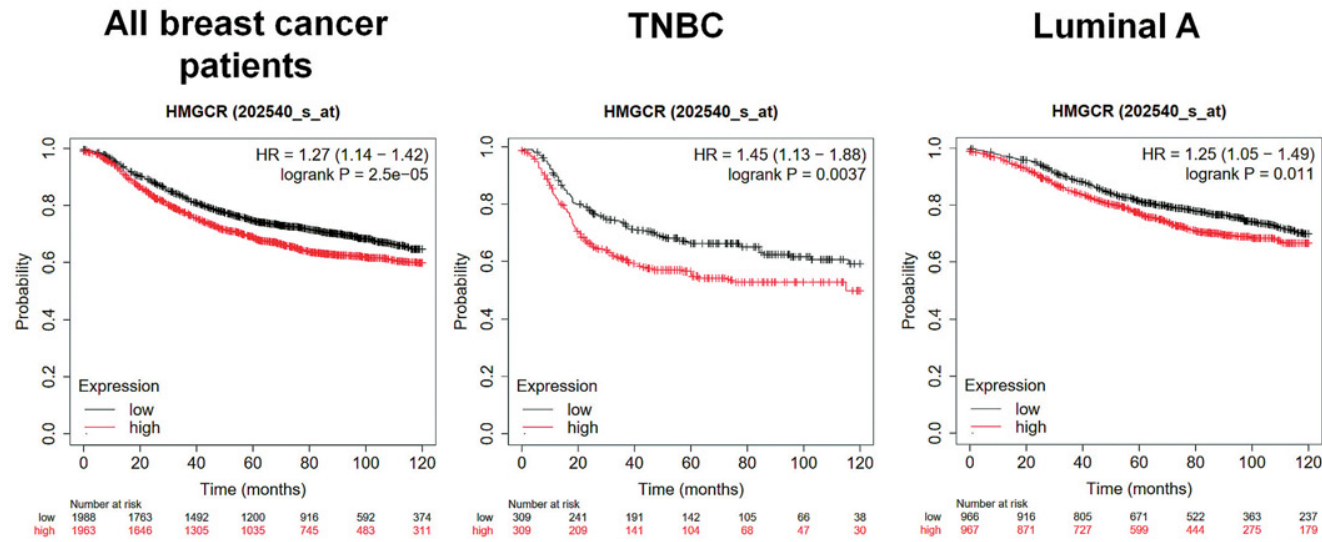

\section{Her2neu-positive}

FIGURE 7. 3-hydroxy-3-methylglutharyl-coenzyme A reductase (HMGCR) expression and recurrence free survival in breast cancer patients. Prognostic value of the HMGCR expression at the mRNA level in breast cancer patients was evaluated using the KMplot database (http://kmplot.com/analysis/), and the results indicate that higher HMGCR expression was associated with worse overall survival in all cohorts of patients.

of $37 \mathrm{kDa}$ in T47D-RR cells or in caspase-9 cleavage accompanied by formation of a 37 and $35 \mathrm{kDa}$ form in MDA-MB-231-RR and Au565-RR cells. Additionally, release of cytochrome $C$ correlated with caspase- 9 activation in the investigated parental and radioresistant breast carcinoma cells. Thus, there were very weak differences in cytochrome $\mathrm{C}$ expression in parental and radioresistant MDAMB-231 cells that resulted in the similar caspase-9 activation with slightly more pronounced cleavage in radioresistant cells. Very low constitutive cytochrome C expression in the parental T47D cells was upregulated at 24 hours after simvastatin treatment only. Radioresistant T47D-RR cells were characterized by more stable release of cytochrome $\mathrm{C}$ after treatment with simvastatin alone, irradiation alone, and especially after combination treatment. Although cytochrome $\mathrm{C}$ expressions were not altered by any kind of treatment in parental and radioresistant Au565 breast carcinoma cells, radioresistant Au565-RR cells demonstrated higher cytochrome $\mathrm{C}$ expression compared to their parental counterparts.

Simvastatin-induced activation of initiator caspases 8 and 9 resulted in the modulation of expression of the executioner caspases 3 and 7. Although both parental and radioresistant MDA-MB-231 cells showed high expression of the total caspase-3, they were deficient in caspase-3 cleavage after all kinds of cell treatment with simvastatin alone, irradiation alone or their combination. Parental T47D breast carcinoma cells revealed comparable results as parental and radioresistant MDA-MB-231 cells. In contrast, radioresistant T47D-RR cells, parental Au565 and radioresistant Au565-RR cells demonstrated very pronounced simvastatin-induced cas- pase- 3 cleavage at 48 and 72 hours after treatment with simvastatin. Irradiation alone was unable to induce cleavage caspase-3, however combination of simvastatin and irradiation led to equal caspase-3 cleavage as observed in simvastatin-treated breast carcinoma cells. Another executioner caspase-7 was fully cleaved in parental and radioresistant MDA-MB-231 and Au565 cells after treatment with simvastatin alone or its combination with irradiation. Since neither caspase-3 nor caspase-7 cleavages were observed in the parental T47D cells, PARP-1 cleavage was also not observed in these cells after any kind of treatment. In contrast, activation of caspases executioners was accompanied by PARP-1 cleavage in parental and radioresistant MDA-MB-231 and Au565 cells, and radioresistant T47D-RR cells after application of simvastatin alone, irradiation alone, and their combination. It is necessary to note that PARP-1 was even cleaved in the cells treated with irradiation alone, though activation of caspases initiators and executioners was not detected. This led to the hypothesis that other mechanisms of cell death such as caspaseindependent apoptosis and autophagy could be implicated in cell killing after cell exposure to irradiation alone or its combination with simvastatin.

Indeed, AIF and Smac/DIABLO expressions were modified by the treatment with simvastatin, irradiation, and their combination. Upregulation of AIF was more pronounced in radioresistant breast carcinoma cells than in their parental counterparts after administration of all treatments. Smac/ DIABLO was weakly expressed in parental and radioresistant T47D cells, but it was increased by simvastatin, irradiation, or their combination in parental and radioresistant MDA-MB-231 and Au565 
cells. Constitutive expression of the apoptosis inhibitor XIAP was enhanced in all radioresistant breast carcinoma cells. However, simvastatin alone or its combination with irradiation decreased XIAP expression. Although XIAP was up-regulated in parental and radioresistant MDA-MB-231 cells after their exposure to ionizing radiation, combination of simvastatin and irradiation did not result in the augmentation of XIAP expression.

Autophagy-related proteins beclin-1 and LC3 $\mathrm{A} / \mathrm{B}$ were also analyzed for their constitutive and treatment-modulated expressions in parental and radioresistant breast carcinoma cells. Beclin-1 did not show any significant dysregulation in response to all treatment approaches. Although LC3 A/B was activated and cleaved after application of simvastatin alone or its combination with irradiation in parental and radioresistant MDA-MB-231 and Au565 cells, parental and radioresistant T47D cells were characterized by LC3 A/B cleavage after cell exposure to all kinds of cell treatment. It is necessary to note that the most prominent up-regulation of LC3 $\mathrm{A} / \mathrm{B}$ expression and enhancement of its cleavage were detected in radioresistant MDA-MB-231-RR and T47D-RR breast carcinoma cells after simvastatin-containing treatment. Thus, LC3 A/B-II/LC3 A/B-I ratio in parental MDA-MB-231 and radioresistant MDA-MB-231-RR cells was $\sim 0,6$ and $\sim 0,5$ after simvastatin treatment at 72 hours, and $\sim 0,6$ and $\sim 0,8$ after combination treatment, respectively; for parental T47D and radioresistant T47D-RR cells: $\sim 0.3$ and $\sim 0.8$ after simvastatin treatment at 72 hours, and $\sim 0,3$ and $\sim 0,8$ after combination treatment, respectively; for parental Au565 and radioresistant Au565-RR cells: 0.2 and $\sim 0.4$ after simvastatin treatment at 72 hours, and $\sim 0,5$ and 0,4 after combination treatment, respectively.

\section{Discussion}

Radiotherapy is an important therapeutic approach used in the management of breast cancer. Although radiotherapy techniques are markedly improved, the problem of radiation resistance of the primary or secondary (relapsed) tumors still exists. ${ }^{4,14}$ It is currently known that radiation resistance can be supported by activated pro-survival intracellular pathways and microenvironmental factors. ${ }^{15,16} \mathrm{~A}$ Western diet containing a lot of fat links not only increased levels of cholesterol in blood but can also be associated with the formation of more aggressive malignant breast tumors in women. ${ }^{15,17}$ Tumor aggressiveness is characterized by reduced cancer cell sensitivity to currently existing therapeutic approaches and inclination for metastatic spread. ${ }^{18-20}$ Since high cholesterol levels could be related to the diminished therapy response and metastatic progression of breast cancer ${ }^{21-23}$, it is assumed that administration of statins can help to improve tumor sensitivity to anti-cancer treatment and attenuate the metastatic cancer cell capabilities.

Simvastatin is one of the widely used statins inhibiting the HMGCR and reducing hypercholesterolemia in patients. Since cholesterol levels can be regulated by the HMGCR, and cholesterolemia can be linked to more aggressive behavior of breast tumors, it is hypothesized that HMGCR can be upregulated in breast cancers with unfavorable clinical outcomes. Indeed, analysis of correlation between HMGCR expression and overall survival in breast cancer patients ${ }^{24}$ has shown that HMGCR overexpression can be associated with reduced overall survival in breast cancer patients independently from the molecular subtypes of the tumors (Figure 7). Accordingly, our data have clearly demonstrated that breast carcinoma cells with confirmed radiation resistance possessed an augmentation of HMGCR expression. It was previously established that HMGCR is implicated in radiation response of melanoma cells, and lipophilic HMGCR inhibitor pitavastatin (Livalo) causes a delay in DNA repair resulting in the persistence of double strand breaks and development of senescence in malignant cells. ${ }^{23}$ Although radiosensitizing and additional anti-tumor effects of the lipophilic statins are previously described ${ }^{22,23,25-29}$, there is only one report demonstrating that simvastatin can sensitize esophageal carcinoma cells to ionizing radiation via inhibition of PI3/Akt pathway. ${ }^{30}$ To our knowledge, there are no publications showing a simvastatin efficacy on cell death and survival of radioresistant breast carcinoma cells. In this study, we have found that simvastatincaused cytotoxic effects were observed in breast carcinoma cells expressing HMGCR, and the levels of their radiation responses did not play the significant roles. While irradiation alone resulted in the HMGCR up-regulation in all parental and radioresistant breast carcinoma cells independently from the constitutive HMGCR levels, combination treatment with simvastatin and ionizing radiation was not accompanied by enhancement of HMGCR in the majority of the investigated carcinoma cells. Only parental hormone receptor-dependent T47D cells lacking constitutive HMGCR expression did not show simvastatin-dependent downregulation of radiation-caused HMGCR expression in the ir- 
radiated cells. We assume that simvastatin was not able to prevent radiation caused HMGCR expression due to the constitutive lack of the target for simvastatin.

Interesting to note, there were no significant differences in cytotoxic cell responses to simvastatin alone and its combination with a clinically relevant dose of irradiation of $2 \mathrm{~Gy}$. Simvastatin alone was equally effective as a combination treatment in both parental and radioresistant breast cancer cells if they expressed HMGCR. However, radiation-caused HMGCR up-regulation in the parental T47D cells did not influence the cytotoxic activities of simvastatin in combination with irradiation. We also cannot exclude that combination of simvastatin with a higher dose of irradiation can more effectively kill breast cancer cells than drug or ionizing radiation alone.

As expected, radioresistant breast carcinoma cells revealed an enhancement of metastasis-associated properties, such as increased migratory abilities and acquisition of more mesenchymal phenotype. Simvastatin treatment caused phenotypic transition of tumor cells between mesenchymal and epithelial states, which was accompanied by the reduction in migratory capabilities of breast carcinoma cells. Hence, it is possible to speculate that metastatic potential of radioresistant cells can be affected by the use of simvastatin.

Although there are several publications reporting on the inhibitory activities of statins on the epithelial-to-mesenchymal transition (EMT) ${ }^{30-34}$, our data additionally provide new evidence that simvastatin can effectively kill radioresistant breast carcinoma cells possessing a mesenchymal phenotype. We, therefore, hypothesize that radioresistant cells can be eliminated by simvastatin from local and/or distant recurrences. This assumption agrees with a clinical observation that statins cannot prevent breast cancer formation but can reduce cancer-related mortality in metastatic breast cancer patients. ${ }^{33}$ It was also shown that lipophilic statins, including simvastatin, can improve a recurrence-free survival in breast cancer patients. ${ }^{33,34} \mathrm{We}$, therefore, suppose that statins alter therapy-resistant breast carcinoma cells and affect carcinoma cell recovery after treatment.

In our study, simvastatin induced cell death in HMGCR-expressing breast carcinoma cells, and we have detected activation of extrinsic, intrinsic, and caspase-independent apoptotic pathways, and autophagy. Although HMGCR-positive breast carcinoma cells demonstrated comparable simvastatin-induced cell death development, apoptosis was differently regulated either with more pronounced involvement of caspase-dependent or caspase-independent pathways. Interesting to note that simvastatin enhanced the expression of autophagyrelated proteins beclin-1 and LC3 in HMGCRnegative and simvastatin-resistant parental T47D cells. However, LC3 activation and cleavage was not very pronounced in parental cells as it was observed in radioresistant T47D cells. Therefore, we suppose that parental cells can be protected from cell death via weak activation of autophagy. ${ }^{35}$ Since we have observed a variety of mechanisms regulating different types of cell death in simvastatin-treated carcinoma cells, it was impossible to detect any unique scenario of breast cancer cell killing after simvastatin treatment. In our opinion, it opens the wider perspectives to use simvastatin as a therapeutic approach to treat breast carcinomas possessing different capabilities for activation of apoptosis or autophagy.

We conclude, radioresistant breast carcinoma cells possessing HMGCR expression accompanied by EMT activation can be successfully killed by simvastatin via mobilizing of a variety of pathways involved in apoptosis and autophagy.

\section{Acknowledgement}

This study was supported by Austrian Science Fund (FWF P29457; FWF I4140), Anniversary Fund of Austrian National Bank (ÖNB 17620), Ingrid Shaker-Nessmann Cancer Research Foundation. Anton Buzdin and Maksim Sorokin were financed by the Russian Foundation for Basic Research Grant 19-29-01108. The research was supported also by the Slovenian Research Agency (ARRS) grant number P3-0003.

\section{References}

1. EU Science Hub. 2020 Cancer incidence and mortality in EU-27 countries. [Internet]. [cited 2021 Jan 22]. Available at: https://ec.europa.eu/jrc/en/ news/2020-cancer-incidence-and-mortality-eu-27-countries

2. Thomas MA, Ochoa LL, Zygmunt TM, Matesa M, Altman MB, GarciaRamirez JL, et al. Accelerated partial breast irradiation: a safe, effective, and convenient early breast cancer treatment option. Mo Med 2015; 112: 379-84. PMID: 26606820

3. Merino T, Tran WT, Czarnota GJ. Re-irradiation for locally recurrent refractory breast cancer. Oncotarget 2015; 6: 35051-62. doi: 10.18632/oncotarget.6036

4. Siglin J, Champ CE, Vakhnenko Y, Anne PR, Simone NL. Radiation therapy for locally recurrent breast cancer. Int J Breast Cancer 2012; 2012: 571946. doi: $10.1155 / 2012 / 571946$ 
5. Fredriksson I, Liljegren G, Arnesson L-G, Emdin SO, Palm-Sjövall M, Fornander T, et al. Local recurrence in the breast after conservative surgery - a study of prognosis and prognostic factors in 391 women. Eur J Cancer 2002; 38: 1860-70. doi: 10.1016/s0959-8049(02)00219-8

6. Alpert TE, Kuerer HM, Arthur DW, Lannin DR, Haffty BG. Ipsilateral breast tumor recurrence after breast conservation therapy: outcomes of salvage mastectomy vs. salvage breast-conserving surgery and prognostic factors for salvage breast preservation. Int J Radiat Oncol Biol Phys 2005; 63: 845 51. doi: 10.1016/j.ijrobp.2005.02.035

7. Huang E, Buchholz TA, Meric F, Krishnamurthy S, Mirza NQ, Ames FC, et al. Classifying local disease recurrences after breast conservation therapy based on location and histology: new primary tumors have more favorable outcomes than true local disease recurrences. Cancer 2002; 95: 2059-67. doi: 10.1002/cncr.10952

8. Bedwinek J. Natural history and management of isolated local-regional recurrence following mastectomy. Semin Radiat Oncol 1994; 4: 260-9. doi: 10.1053/SRAO00400260

9. Belkacemi Y, Hanna NE, Besnard C, Majdoul S, Gligorov J. Local and regional breast cancer recurrences: salvage therapy options in the new era of molecular subtypes. Front Oncol 2018; 8: 112. doi: 10.3389/fonc.2018.00112

10. Matusewicz L, Czogalla A, Sikorski AF. Attempts to use statins in cancer therapy: an update. Tumor Biol 2020; 42: 1010428320941760. doi: $10.1177 / 1010428320941760$

11. Skvortsova I, Skvortsov S, Popper BA, Haidenberger A, Saurer M, Gunkel AR, et al. Rituximab enhances radiation-triggered apoptosis in Non-Hodgkin's lymphoma cells via caspase-dependent and-independent mechanisms. $J$ Radiat Res 2006; 47: 183-96. doi: 10.1269/jrr.47.183

12. Skvortsova I, Skvortsov S, Haidenberger A, Devries A, Nevinny-Stickel M, Saurer $\mathrm{M}$, et al. Effects of paclitaxel and docetaxel on EGFR-expressing human carcinoma cells under normoxic versus hypoxic conditions in vitro. $J$ Chemother 2004; 16: 372-80. doi: 10.1179/joc.2004.16.4.372

13. Skvortsov $\mathrm{S}$, Sarg B, Lindner $\mathrm{H}$, Lukas $\mathrm{P}$, Hilbe $\mathrm{W}$, Zwierzina $\mathrm{H}$, et al. Cetuximab inhibits thymidylate synthase in colorectal cells expressing epidermal growth factor receptor. Proteomics Clin Appl 2008; 2: 908-14. doi 10.1002/prca. 200780034

14. Choi J, Yoon YN, Kim N, Park CS, Seol H, Park I-C, et al. Predicting radiation resistance in breast cancer with expression status of phosphorylated S6K1. Sci Rep 2020; 10: 641. doi: 10.1038/s41598-020-57496-8

15. Garcia-Estevez L, Moreno-Bueno G. Updating the role of obesity and cholesterol in breast cancer. Breast Cancer Res 2019; 21: 35. doi: 10.1186/ s13058-019-1124-1

16. Hirata $E$, Sahai E. Tumor microenvironment and differential responses to therapy. Cold Spring Harb Perspect Med 2017; 7: a026781. doi: 10.1101/ cshperspect.a026781

17. Bjarnadottir O, Feldt M, Inasu M, Bendahl PO, Elebro K, Kimbung S, et al. Statin use, HMGCR expression, and breast cancer survival - The Malmo Die and Cancer Study. Sci Rep 2020; 10: 558. doi: 10.1038/s41598-019-57323-9

18. Arnold CR, Mangesius J, Skvortsova II, Ganswindt U. The role of cancer stem cells in radiation resistance. Front Oncol 2020; 10: 164. doi: 10.3389/ fonc.2020.00164

19. Steinbichler TB, Dudás J, Skvortsov $S$, Ganswindt U, Riechelmann $H_{4}$ Skvortsova I-I. Therapy resistance mediated by cancer stem cells. Semin Cancer Biol 2018; 53: 156-67. doi: 10.1016/j.semcancer.2018.11.006

20. Steinbichler TB, Savic D, Dudás J, Kvitsaridze I, Skvortsov S, Riechelmann $H_{\text {, }}$ et al. Cancer stem cells and their unique role in metastatic spread. Semin Cancer Biol 2020; 60: 148-56. doi: 10.1016/j.semcancer.2019.09.007

21. Wu QJ, Tu C, Li YY, Zhu J, Qian KQ, Li WJ, et al. Statin use and breast cance survival and risk: a systematic review and meta-analysis. Oncotarget 2015; 6: 42988-3004. doi: 10.18632/oncotarget.5557

22. Efimova E V, Ricco N, Labay E, Mauceri HJ, Flor AC, Ramamurthy A, et al. HMG-CoA reductase inhibition delays DNA repair and promotes senescence after tumor irradiation. Mol Cancer Ther 2018; 17: 407-18. doi: 10.1158/1535-7163.MCT-17-0288

23. Longo J, van Leeuwen JE, Elbaz M, Branchard E, Penn LZ. Statins as anticancer agents in the era of precision medicine. Clin Cancer Res 2020; 26 5791-800. doi: 10.1158/1078-0432.CCR-20-1967

24. Kaplan-Meier Plotter. Breast cancer. [Internet]. [cited 2021 Jan 22]. Available at: https://kmplot.com/analysis/
25. Ricco N, Flor A, Wolfgeher D, Efimova EV, Ramamurthy A, Appelbe OK, et al. Mevalonate pathway activity as a determinant of radiation sensitivity in head and neck cancer. Mol Oncol 2019; 13: 1927-43. doi: 10.1002/18780261.12535

26. Wolfe AR, Debeb BG, Lacerda L, Larson R, Bambhroliya A, Huang X, et al. Simvastatin prevents triple-negative breast cancer metastasis in pre-clinical models through regulation of FOXO3a. Breast Cancer Res Treat 2015; 154: 495-508. doi: 10.1007/s10549-015-3645-3

27. Wolfe AR, Woodward WA. Breast cancer stem cell correlates as predicative factors for radiation therapy. Semin Radiat Oncol 2015; 25: 251-9. doi: 10.1016/j.semradonc.2015.05.009

28. Van Wyhe RD, Rahal OM, Woodward WA. Effect of statins on breast cancer recurrence and mortality: a review. Breast Cancer 2017; 9: 559-65. doi: 10.2147/BCTT.S148080

29. Rahal OM, Woodward WA. Cholesterol and radiosensitivity. Curr Breast Cancer Rep 2016; 8: 32-9. doi: 10.1007/s12609-016-0202-y

30. Jin Y, Xu K, Chen Q, Wang B, Pan J, Huang S, et al. Simvastatin inhibits the development of radioresistant esophageal cancer cells by increasing the radiosensitivity and reversing EMT process via the PTEN-PI3K/AKT pathway. Exp Cell Res 2018; 362: 362-9. doi: 10.1016/j.yexcr.2017.11.037

31. Xie F, Liu J, Li C, Zhao Y. Simvastatin blocks TGF- $\beta 1$-induced epithelialmesenchymal transition in human prostate cancer cells. Oncol Lett 2016; 11: 3377-83. doi: 10.3892/ol.2016.4404

32. Kato S, Liberona MF, Cerda-Infante J, Sánchez M, Henríquez J, Bizama C, et al. Simvastatin interferes with cancer "stem-cell" plasticity reducing metastasis in ovarian cancer. Endocr Relat Cancer 2018; 25: 821-36. doi: 10.1530/ ERC-18-0132

33. Ahern TP, Pedersen L, Tarp M, Cronin-Fenton DP, Garne JP, Silliman RA, et al. Statin prescriptions and breast cancer recurrence risk: a Danish nationwide prospective cohort study. J Natl Cancer Inst 2011; 103: 1461-8. doi: 10.1093/jnci/djr291

34. Manthravadi S, Shrestha A, Madhusudhana S. Impact of statin use on cancer recurrence and mortality in breast cancer: a systematic review and meta-analysis. Int J cancer 2016; 139: 1281-8. doi: 10.1002/ijc.30185

35. Bhutia SK, Mukhopadhyay S, Sinha N, Das DN, Panda PK, Patra SK, et al. Autophagy: cancer's friend or foe? Adv Cancer Res 2013; 118: 61-95. doi: 10.1016/B978-0-12-407173-5.00003-0 